\title{
Biological control of sugarcane caterpillar (Diatraea saccharalis) using interval mathematical models
}

\author{
José Renato Campos *, Edvaldo Assunção ${ }^{\dagger}$, Geraldo Nunes Silva $\ddagger$ and Weldon Alexander Lodwick $\S$ \\ * Area of Sciences \\ Federal Institute of Education, Science and Technology of São Paulo, Votuporanga, SP, Brazil \\ jrcifsp@ifsp.edu.br, jrcifsp@gmail.com \\ $\dagger$ Department of Electrical Engineering \\ UNESP - Univ Estadual Paulista, Ilha Solteira, SP, Brazil \\ edvaldo@dee.feis.unesp.br \\ $\ddagger$ Department of Applied Mathematics \\ UNESP - Univ Estadual Paulista, São José do Rio Preto, SP, Brazil \\ gsilva@ibilce.unesp.br \\ $\S$ Department of Mathematical and Statistical Sciences \\ University of Colorado, Denver, Colorado, USA \\ weldon.lodwick@ucdenver.edu
}

Received: 20 August 2015, accepted: 23 April 2016, published: 2 May 2016

\begin{abstract}
Biological control is a sustainable agricultural practice that was introduced to improve crop yields and has been highlighted among the various pest control techniques. However, real mathematical models that describe biological control models can have error measurements or even incorporate lack of information. In these cases, intervals may be feasible for indicating the lack of information or even measurement errors. Therefore, we consider interval mathematical models to represent the biological control problem. Specifically, in the present paper, we illustrate the solution of a discrete-time interval optimal control problem for a practical application in biological control. To solve the problem, we use single-level constrained interval arithmetic [9] and the dynamic programming technique [3]
\end{abstract}

along with the idea proposed in [23] for the solution of the interval problem.

Keywords-Interval optimal control problem; interval mathematical models; single-level constrained interval arithmetic; dynamic programming; biological control.

\section{INTRODUCTION}

Sugarcane culture plays an important role in the Brazilian economy. It is estimated that the country has more than 8 million hectares of cultivated area [1] and that sugarcane is responsible for over 4.5 million jobs [38]. In addition to the production of sugar, ethanol and various other byproducts, it is also used to produce electricity with the use 
José Renato Campos et al., Biological control of sugarcane caterpillar ...

of biomass (bagasse and straw). Thus, sustainable management of this culture is fundamental. Among the various types of management that can be implemented (control of pests and weeds, soil handling, etc.) and the various methods of manufacture (biological control, use of insecticide and herbicide, manual and mechanical control, etc.), pest control through biological control stands out.

Biological control is sustainable because it does not affect the environment. For the culture of sugarcane, the control involves the caterpillar and wasp. The caterpillar (Diatraea Saccharalis) is an insect that causes damage to the crop, and its natural predator, Cotesia Flavipes, is a wasp that deposits its eggs on the caterpillar and inhibits the development of the caterpillar. Hence, the caterpillar dies without completing its life cycle and without causing economic loss to the crop.

The spread of the caterpillar can cause damage to the crop such as weight loss and reduction in germination, leading to the death of germinating plants, which directly reflects on the costs of production. Thus, the biological control of pests is a good alternative to the feasibility of such crops for the country. In addition, the biological control process is part of the integrated crop protection [11] that is a benchmark for sustainable farming practices.

Control theory study began in the USA in the 1930 s with studies of problems in electrical engineering and mechanical engineering [8]. In the 1950s, with optimization methods developed by Bellmann in 1957 (see [2]) and Pontryagin in 1958 (see [29], [30]), modern control theory or optimal control theory was born. Such theory brought advances in several areas such as Agriculture, Biology, Economics, Engineering and Medicine.

In Agriculture or Biology, deterministic optimal control problems are widely studied, and some biomathematical models illustrating deterministic models can be found in [7], [15], [16], [19], [37]. In these studies, conventional models were assumed with fixed coefficients.

For problems with uncertain parameters, the optimal control problem usually utilizes stochasticity
[4], [16] or, more recently, fuzzy set theory [12], [10], [28]. In the two cases, the coefficients are viewed as random variables or as fuzzy sets, and it is assumed that their probability distributions or membership functions, respectively, are known.

In biological problems, uncertainty arises frequently because it is inherent to the determination of biological data; for example, uncertainty arises due to measurement errors, inaccuracies in the equipment, climatic factors, and lack of specification, among many others. Thus, we propose interval uncertainty to describe the uncertainty in obtaining data in biological problems. We can represent a parameter of the model, such as the mortality rate of predators, as an interval. This is relevant because we can model an environment with several variations in the mortality rates of predators and not have to consider a unique rate for all the predators, especially if this information has been obtained imprecisely.

Optimal control problems involving uncertain systems are described in [6], [13], [14], [39]. However, in these approaches, the functional is a real number and thus differs from the approach proposed in this paper. Additionally, the problem discussed here does not include state feedback. References on control problems that present interval uncertainty but still differ from that proposed in this paper can be found in [20], [17], [32].

Thus, in this work, we consider a new kind of problem called the interval optimal control problem. The interval arithmetic used in this approach is described in [9], [21], [22] and is different from the standard interval arithmetic proposed in [24]. To solve the interval optimal control problem, we choose single-level constrained interval arithmetic [9] because it eliminates certain problems related to other types of interval arithmetic, such as the existence of the additive inverse or the distributive law property. Single-level constrained interval arithmetic also has properties closer to the space of real numbers. Therefore, we study the discrete time interval optimal control problem with the interval initial condition or interval parameters in the dynamic equation. 
José Renato Campos et al., Biological control of sugarcane caterpillar ...

The paper is arranged as follows. Section II presents the application in Biology, and some biological aspects will be demonstrated. We also present the deterministic and interval optimal control problem. In Section III, we present the solutions of the discrete time interval optimal control problems previously proposed. The discussion of the results is provided in Section IV.

\section{THE BIOMATHEMATICAL MODEL}

The biological situation studied is a problem encountered in sugarcane culture. According to Silva and Bergamasco [35], the environmental management of sugarcane culture requires performance prediction in production and environmental risk at various levels of control in sugarcane production because manipulation of the soil, planting depth and density, pest and diseases, among other factors, and biological control have proven to be effective in operational management of the culture.

Thus, the problem studied corresponds to a model of competition between the wasp (Cotesia Flavipes) and the caterpillar (Diatraea Saccharalis) in terms of sugarcane, represented using the LotkaVolterra two-species model.

Tusset and Rafikov in [37] ran a simulation of the dynamics of the system without application control and showed that the system begins to stabilize at 350 days and that during this period, economic losses are experienced. Thus, we need to apply control in previous periods, and the application of control corresponds to the introduction of wasps in sugarcane culture.

Tusset and Rafikov [37] solve the continuous deterministic optimal control problem using the Riccati equation. Campos [7] also solved the deterministic and discrete problem using dynamic programming, and the results are similar for the two approaches.

The goal here is to present the interval optimal control problem and solve the biological control problem encountered in sugarcane culture. We analyze the biological situation and describe the biomathematical model. According to Tusset and Rafikov [37], the Lotka-Volterra two-species model used in the problem of sugarcane culture is given by

$$
\left\{\begin{array}{l}
\dot{x}=x(a-\gamma x-c y) \\
\dot{y}=y(-d+r x)+u^{*}+u
\end{array},\right.
$$

where $x(t)$ is the number of preys and $y(t)$ is the number of predators for $t \geq 0$. Here, $u^{*}$ is the control that carries the system to the desired equilibrium point, and $u$ is the control that stabilizes the system at this point.

The dynamic model (1) is a Lotka-Volterra model for the case of the caterpillar that is the sugarcane parasitoid, where the coefficient $a$ represents the interspecific growth of the preys, the coefficient $d$ represents the mortality of the predators, $c$ represents the capture rate, $r$ is the maximum rate of growth of the predator population, and $\gamma$ is the self-inhibition coefficient of growth of the preys due to restriction of food.

According to [37], the parameter $a$ is calculated assuming the absence of predators in (1). Then, we obtain

$$
\dot{x}=x(a-\gamma x),
$$

where we suppose that $\gamma=a / k$. Solving the differential equation (2) and isolating the value of the parameter $a$, we obtain

$$
a=-\frac{1}{t}\left[\ln \left(\frac{\frac{k-x}{x}}{\frac{k-x^{0}}{x^{0}}}\right)\right] .
$$

Assuming $k=25000$ and considering that the caterpillar lives on average 70 days and after mating lays on average 300 eggs (see [27]), we find that $t=70$ days with $x(70)=300$ caterpillars per hectare. Assuming an initial number of preys equal to $x^{0}=2$ caterpillars per hectare, it follows that the interspecific growth of the caterpillar is $a=0.0716$ caterpillars per hectare per day.

The calculation of the other parameters of the dynamic equation of problem (1) can be found in [37], following a similar analysis.

Thus, in this work, we obtain the numerical coefficients $a, \gamma, c, d$ and $r$ in [37] as well as the expression for the functional of the optimal control 
José Renato Campos et al., Biological control of sugarcane caterpillar ...

problem. The problem proposed in [37] with a quadratic objective function subject to nonlinear restrictions is given by

$\min C=\frac{1}{2} \int_{0}^{t_{f}} 8\left(x-x^{*}\right)^{2}+0.2841\left(y-y^{*}\right)^{2}+u^{2} d t$ subject to

$$
\left\{\begin{array}{l}
\dot{x}=x(0.0716-0.0000029 x-0.0000464 y) \\
\dot{y}=y(-1+0.000520235 x)+u^{*}+u
\end{array},\right.
$$

where $t_{f}$ is the final time, the initial conditions are $x_{0}=5000$ and $y_{0}=1500$, and the final conditions are the desired equilibrium point $\left(x^{*}, y^{*}\right)$. From a biological point of view, Segato et al. [33] show that when the number of preys (Diatraea Saccharalis) reaches 5000 caterpillars per hectare, application of control $u$ corresponds to the release of predators (Cotesia Flavipes).

The calculations for the numerical coefficients of the states $x$ and $y$ in the functional of problem (3) are extensive and can be found in [37]; such calculations are based on [34], [31]. Furthermore, Tusset and Rafikov consider in [37] a positive semidefinite and symmetric quadratic functional in order to take the system to the desired equilibrium point the fastest way possible when considering only small oscillations in the path of the system. This is important for the biological control problem studied.

To solve problem (3), Tusset and Rafikov in [37] considered a problem with a linear dynamic equation. The linearization of the model is feasible because we suppose that the linear and nonlinear dynamic system behaviors are qualitatively equivalent in the vicinity of the equilibrium point (see [25], Grobman-Hartman Theorem). Thus, the dynamic equation of problem (3) is linearized (see [25]) assuming that the initial conditions are near the equilibrium point $(2000,1418.10)$. In a real system, this is possible when we apply a value several times that of the control.

According to Botelho and Macedo [5] for the sugarcane crop, greater than or equal to 2500 caterpillars per hectare causes damage to the culture. We fix $x^{*}=2000$ (a value that does not cause damage) and hence obtain the value $y^{*}$ using the equation $f\left(x^{*}, y^{*}\right)=0$, where $f(x, y)=0.0716-0.0000029 x-0.0000464 y$. Therefore, the desired equilibrium point for the prey and the predator is represented by $\left(x^{*}, y^{*}\right)=$ $(2000,1418.10)$ and used in the final condition of the problem.

Finally, the optimal control problem with a quadratic objective function subject to linear restrictions proposed in [37] is given by

$$
\min C=\frac{1}{2} \int_{0}^{t_{f}} 8 z_{1}^{2}+0.2841 z_{2}^{2}+u^{2} d t
$$

subject to

$$
\dot{z}=\left[\begin{array}{cc}
-0.0058 & -0.0928 \\
0.7386 & 0.0405
\end{array}\right] z+\left[\begin{array}{l}
0 \\
1
\end{array}\right] u,
$$

with initial conditions $z_{10}=3000$ and $z_{20}=$ 80.17 due to translation to the equilibrium point. Note that $z=\left(z_{1}, z_{2}\right)^{T}=\left(x-x^{*}, y-y^{*}\right)^{T}$, where $z$ is the translation of the point of equilibrium $\left(x^{*}, y^{*}\right)$ to the origin and $T$ denotes the transposed vector. In particular, the change in coordinates to problem (4) is performed assuming that we are close to the fixed point; furthermore, the change in coordinates facilitates the computational implementation.

To find the solution of the problem of biological control of the sugarcane caterpillar (4) with a discrete dynamic programming method, Campos [7] discretized problem (4).

The discrete model (and match) proposed in [7] is

$$
\min C=\frac{h}{2} \sum_{k=0}^{N} 8 z_{1 k}^{2}+0.284 z_{2 k}^{2}+u_{k}^{2}
$$

subject to

$z_{k+1}=\left[\begin{array}{cc}0.960 & -0.093 \\ 0.743 & 1.006\end{array}\right] z_{k}+\left[\begin{array}{c}-0.047 \\ 1.009\end{array}\right] u_{k}$ 
José Renato Campos et al., Biological control of sugarcane caterpillar ...

where $z_{k}=\left(z_{1 k}, z_{2 k}\right)^{T}$ and the initial conditions are $z_{10}=3000$ and $z_{20}=80.17$. Here, $k$ denotes the discrete iterations in days for the problem. Furthermore, for problem (5), the simulation period equals $N=18$ days, and hence, $t_{f}=h N=18$ days.

Campos in [7] used the zero-order hold method (function $c 2 d$ in MATLAB 7.4) to discretize the dynamic equation of problem (4). Thus, the zeroorder hold method provides an exact match between the continuous dynamic system of problem (4) and the discrete dynamic system of problem (5). For the biological analysis of the optimal control problem, we are assuming that the control decision $u_{k}$, introduction of predators, occurs only once a day.

The discretization of the functional of problem (4) introduces an error because it is approximated using a numerical quadrature. However, the error in the discretization of the functional does not change the behavior of the dynamic equations of problems (4) and (5). Furthermore, the weight assigned to the coefficient of control $u_{k}$ in the functional of problem (5) can be modified and adapted according to the costs involved in the operations.

Next, we illustrate the formulation of interval control problems for two distinct situations. The first involves the problem with the interval initial condition. The second formulation considers an interval coefficient in the dynamic equation.

\section{A. Uncertainty in the Initial Condition}

Suppose that the model (5) uses the interval initial condition because we consider there to be inaccurate information in the data. We use an interval initial condition of $Z_{10}=[2970,3030]$, which represents an error of $2 \%$. The second initial condition used is $Z_{20}=$ 80.17 and represents a degenerate interval.

Therefore, the problem with the interval initial condition is described below. It is given by subject to

$$
\left\{\begin{array}{l}
Z_{1 k+1}=0.960 \otimes Z_{1 k} \ominus 0.093 \otimes Z_{2 k} \ominus 0.047 \otimes U_{k} \\
Z_{2 k+1}=0.743 \otimes Z_{1 k} \oplus 1.006 \otimes Z_{2 k} \oplus 1.009 \otimes U_{k}
\end{array}\right.
$$

where $Z_{1 k}, Z_{2 k}, U_{k}$ and $\mathbf{C}$ are intervals and the initial conditions are $Z_{10}=[2970,3030]$ and $Z_{20}=80.17$. For the interval problem, the symbols $\oplus, \ominus, \otimes$ and $\oslash$ represent the sum, subtraction, multiplication and division of intervals, respectively, according to single-level constrained interval arithmetic. This model is presented in [7]; however, here it is presented as an interval problem. In particular, the initial condition is also an interval. Problem (6) is called the interval optimal control problem. Furthermore, we emphasize that the functional is an interval and that its optimality is given by the order relation of single-level constrained interval arithmetic (see [18]). According to Leal [18], given two intervals $A=[\underline{a}, \bar{a}]$ and $B=[\underline{b}, \bar{b}]$, the order relation between them is given by

$$
A \leq_{S L} B \text { iff } A(\lambda) \leq B(\lambda) \text { for all } \lambda \in[0,1],
$$

where $\leq_{S L}$ denotes the inequality between intervals according to single-level constrained interval arithmetic and $A(\lambda)$ and $B(\lambda)$ are the convex constraint functions associated with $A$ and $B$, respectively. Note that $A(\lambda)=(1-\lambda) \underline{a}+\lambda \bar{a}, \quad 0 \leq \lambda \leq 1$.

Initially, the interval optimal control problem (6) can be transformed into a real classic problem using single-level constrained interval arithmetic. Thus, the interval optimal control problem (6), rewritten as the single-level constrained interval arithmetic [9], is given by

$$
\min C=\frac{h}{2} \sum_{k=0}^{N} 8 Z_{1 k}^{2}(\lambda)+0.284 Z_{2 k}^{2}(\lambda)+U_{k}^{2}(\lambda)
$$

subject to

$\min \mathbf{C}=\frac{h}{2} \otimes \sum_{k=0}^{N} 8 \otimes Z_{1 k}^{2} \oplus 0.284 \otimes Z_{2 k}^{2} \oplus U_{k}^{2} \quad Z_{k+1}(\lambda)=\left[\begin{array}{cc}0.960 & -0.093 \\ 0.743 & 1.006\end{array}\right] Z_{k}(\lambda)+\left[\begin{array}{c}-0.047 \\ 1.009\end{array}\right] U_{k}(\lambda)$, 
José Renato Campos et al., Biological control of sugarcane caterpillar ...

where $Z_{k}(\lambda)=\left(Z_{1 k}(\lambda), Z_{2 k}(\lambda)\right)^{T}$ and the initial conditions are $Z_{10}(\lambda)=2970+60 \lambda$ and $Z_{20}(\lambda)=$ 80.17, $0 \leq \lambda \leq 1$. Here, $Z_{k}(\lambda)$ and $U_{k}(\lambda)$ are the convex constraint functions associated with intervals $Z_{k}$ and $U_{k}$, respectively. Furthermore, we also suppose $Z_{k}(\lambda)$ and $U_{k}(\lambda)$ to have the appropriate dimensions.

Now, problem (7) is a classic optimal control problem for all fixed $\lambda \in[0,1]$. Therefore, we use dynamic programming as our solution technique for the discrete time optimal control problem. The advantage of dynamic programming is that it determines the optimal solution of a multistage problem by breaking it into stages, where each stage is a subproblem. Solving a subproblem is a simpler task in terms of calculation than dealing with all the stages simultaneously. Moreover, a dynamic programming model is a recursive equation that links the different stages of the problem, ensuring that the optimal solution at each stage is also optimal for the entire problem (see [36]). Details on dynamic programming can be found in [3].

Finally, we solve problem (7) for all fixed $\lambda \in$ $[0,1]$, and we present the solution in the interval space in accordance with the ideas proposed in [9] and [23], i.e., we return the solution to the interval space using the minimum and maximum of the values obtained for each stage of the problem, provided that the minimum and maximum exist.

\section{B. Uncertainty in the Dynamic Equation}

For the interval problem with uncertainty in the dynamic equation, we consider again the biomathematical model (5) described previously. Suppose that, due to some biological factors, the first parameter of the first dynamic equation is an interval. Specifically, consider that due to some inaccuracy in obtaining the data for the model, the interval optimal control problem represents the first parameter of the dynamic equation as an interval, that is, the value 0.960 is substituted by the interval $[0.760,1.160]$. This interval represents $41.67 \%$ of the error in relation to the deterministic value.

Therefore, the interval optimal control problem is $\min \mathbf{C}=\frac{h}{2} \otimes \sum_{k=0}^{N} 8 \otimes Z_{1 k}^{2} \oplus 0.284 \otimes Z_{2 k}^{2} \oplus U_{k}^{2}$

subject to

$\left\{\begin{array}{l}Z_{1 k+1}=[0.760,1.160] \otimes Z_{1 k} \ominus 0.093 \otimes Z_{2 k} \ominus 0.047 \otimes U_{k} \\ Z_{2 k+1}=0.743 \otimes Z_{1 k} \oplus 1.006 \otimes Z_{2 k} \oplus 1.009 \otimes U_{k}\end{array}\right.$

where $Z_{1 k}, Z_{2 k}, U_{k}$ and $\mathbf{C}$ are intervals and the initial conditions are $Z_{10}=3000$ and $Z_{20}=$ 80.17 (degenerate intervals) due to translation of the equilibrium point.

Similar to Subsection II-A, we rewrite the interval problem according to single-level constrained interval arithmetic [9]. We then solve the corresponding problem using dynamic programming [3]. According to the methodology proposed in [23] and [9], we find the solution interval.

The numerical solution to the problems (6) and (8) will be presented in the next section.

\section{NUMERICAL ANALYSIS AND SIMULATIONS}

The implementation and adaptation of the dynamic programming algorithm to solve problems (6) and (8) were performed using MATLAB 7.4. Furthermore, problems (6) and (8) were solved using a microcomputer with a Dual-Core AMD E 300 processor and $3 \mathrm{~GB}$ of memory. For the interval problems, we chose $N=18$ days. The computational time to solve problem (6) was approximately 4.5 minutes, and the computational time required to solve problem (8) was approximately 26 minutes.

The interval cost found in the solution is called the optimal interval cost. The interval state obtained is called the optimal interval state, and the interval control obtained for each iteration in the interval optimal control problem is called the optimal interval control.

The following figures represent the numerical results of problems (6) and (8). The deterministic and discrete solutions are also introduced in the figures. In the solutions presented, the translation of the solution has been reversed. In addition, the points representing the deterministic and interval 
José Renato Campos et al., Biological control of sugarcane caterpillar ...

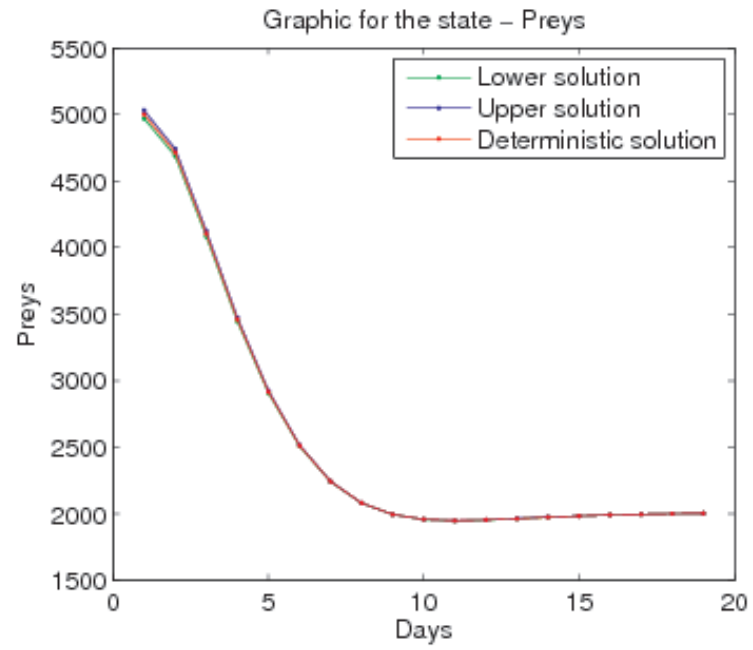

Fig. 1. Preys for problem 6.

solutions to the problem are connected by line segments for facilitating the visualization of the temporal evolution. The solutions given by the minimum and maximum values correspond to the optimal interval solutions.

Graphical solutions are provided for the situations described in problem (6). Figure 11 illustrates the number of preys for the problem with uncertainty in the initial condition. Figure 2 illustrates the number of predators for the same problem. The predators are introduced in Figure 3. and the negative values that appear in the figure correspond to the number of predators that should be removed using some sustainable agricultural practice.

The optimal cost of the deterministic problem is $1.3716 \times 10^{8}$. The optimal interval cost of problem (6) is $\left[1.3443 \times 10^{8}, 1.3992 \times 10^{8}\right]$. Thus, the interval uncertainty inserted in the initial condition of the problem results in a variation in the cost of approximately $4.00 \%$ compared with the deterministic solution.

The graphical solution to problem $(8)$ is presented below. Figure 4 illustrates the number of preys for this problem. Figure 5 illustrates the number of predators for (8). The values of the control variable are presented in Figure 6.

The optimal interval cost of problem (8) is

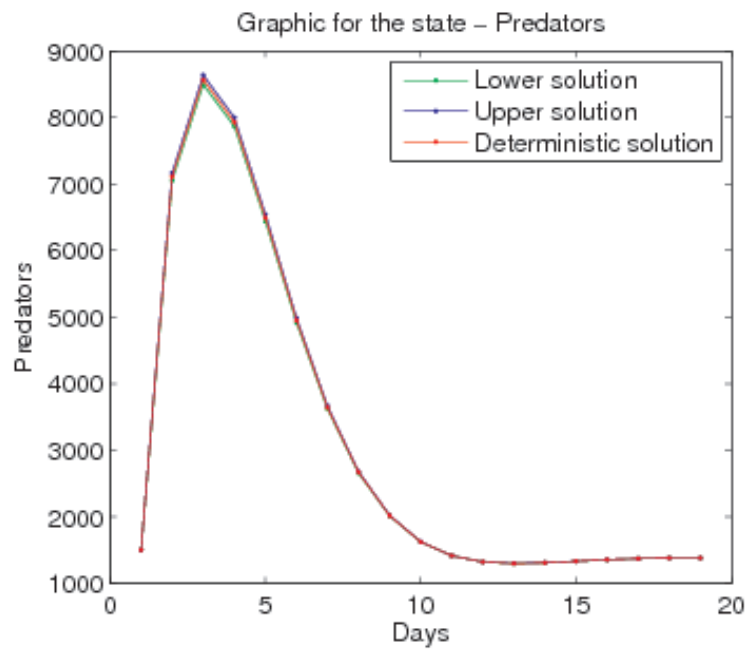

Fig. 2. Predators for problem 6.

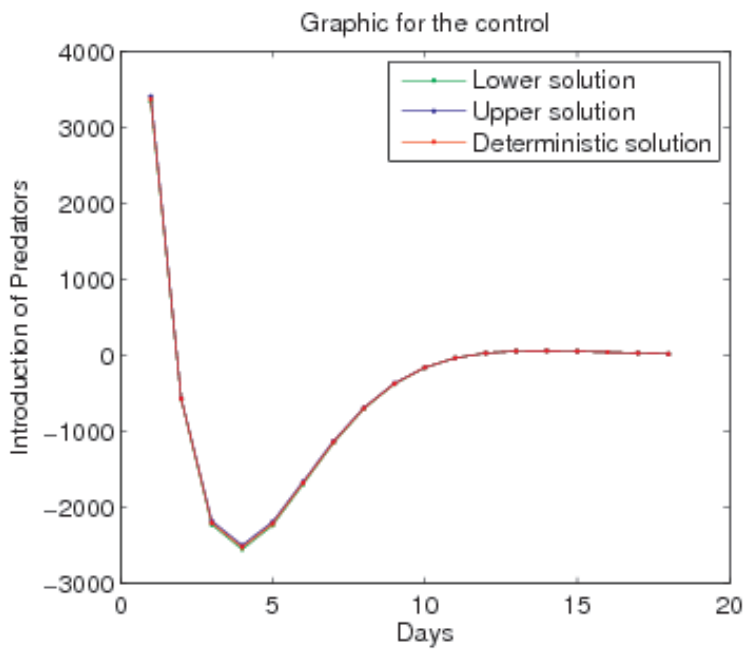

Fig. 3. Introduction of predators for problem (6).

$\left[7.8523 \times 10^{7}, 2.7181 \times 10^{8}\right]$. The uncertainty introduced into the dynamic equation generated a variation of approximately $140.92 \%$ in the functional in relation to the deterministic solution.

Remark 3.1: The solutions of the interval problems (6) and (8) converge to the desired equilibrium point. The interval solutions converge to the desired equilibrium point if the distance between them tends to zero according to the definition of the distance between intervals given by [9]. Thus, the approximate interval $X$ to $x^{*}$ means that the 
José Renato Campos et al., Biological control of sugarcane caterpillar ...

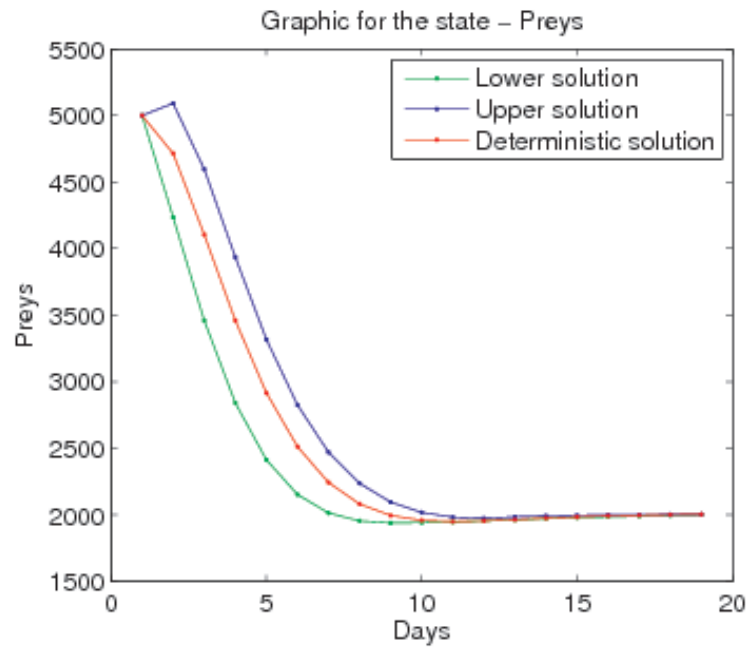

Fig. 4. Preys for problem 8.

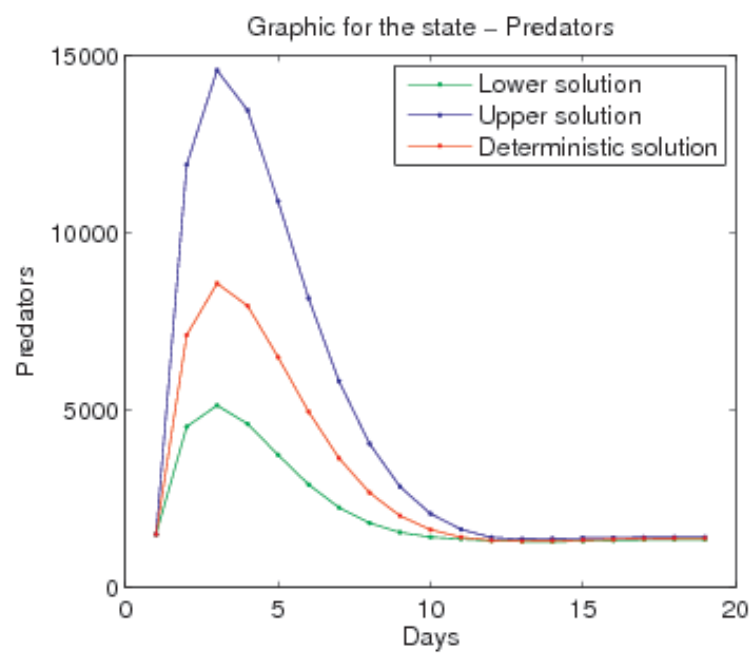

Fig. 5. Predators for problem (8).

distance between them, given by $\max _{0 \leq \lambda \leq 1}\left|X(\lambda)-x^{*}\right|$ where $X(\lambda)$ is a convex constraint function associated with $X$, tends to zero. Further, analyzing the interval problems (6) and (8) according to the associated convex constraint functions (see, for example, problem (7)), we have that the corresponding optimal control problems are classical optimal control problems for all fixed $\lambda \in[0,1]$ and satisfy the stability criterion (see [26], [3]) for optimal control problems with quadratic functional and linear constraints.

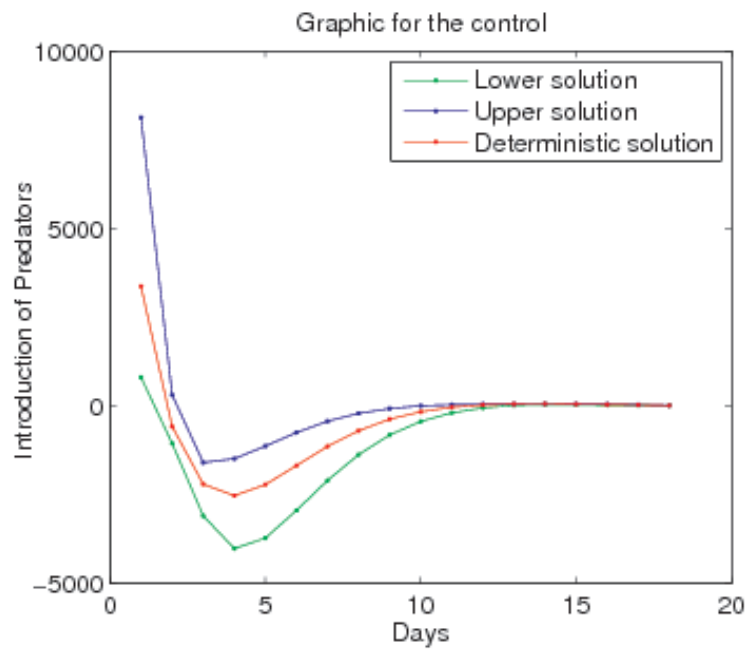

Fig. 6. Introduction of predators for problem (8).

Remark 3.2: Other interval optimal control problems can be investigated, such as the problem with interval initial conditions and interval parameters in the interval dynamic equation. Thus, considering the interval optimal control problem given by

$\min \mathbf{C}=\frac{h}{2} \otimes \sum_{k=0}^{N} 8 \otimes Z_{1 k}^{2} \oplus 0.284 \otimes Z_{2 k}^{2} \oplus U_{k}^{2}$ subject to

$\left\{Z_{1 k+1}=[0.760,1.160] \otimes Z_{1 k} \ominus 0.093 \otimes Z_{2 k} \ominus 0.047 \otimes U_{k}\right.$ $\left\{Z_{2 k+1}=0.743 \otimes Z_{1 k} \oplus 1.006 \otimes Z_{2 k} \oplus 1.009 \otimes U_{k}\right.$

where $Z_{1 k}, Z_{2 k}, U_{k}$ and $\mathbf{C}$ are intervals and the interval initial conditions are $Z_{10}=[2970,3030]$ and $Z_{20}=80.17$, we have that the optimal interval cost is given by $\left[7.6959 \times 10^{7}, 2.7729 \times 10^{8}\right]$. Furthermore, the solution of the interval problem (9) shows basically the same qualitative behavior as that of the solution of the interval problem (8).

\section{DisCUSSION OF THE RESUlTS}

In the problems studied, the initial condition or the dynamic equation has intervals because the data are generally inaccurate and may be represented by interval uncertainty. Consequently, 
José Renato Campos et al., Biological control of sugarcane caterpillar ...

this implies a variation in the functional, state and control at each iteration (cost, state and control represented by intervals). The decision maker should consider whether it is feasible to run the model for the values obtained in these intervals.

Therefore, to analyze if the number of preys or predators achieves the minimum or maximum values is an important question in the decisionmaking process of a manager because it can lead to financial loss and environmental damage. Furthermore, the analysis of interval costs is also very important for the company.

We now emphasize some points from the solutions obtained previously.

\section{A. Analysis of the interval problem (6)}

In the solution presented for the interval problem (6), we found consistency with the deterministic results as can be seen from Figures 1, 2 and 3. The behaviors of the interval state variable and interval control variable are also quite regular and in accordance with the variation of the deterministic solution. The extremes of the intervals of the state interval solutions $X$ and $Y$ approached the desired value, as was observed with the interval control $U$. Therefore, the decision maker obtains values close to those found for the deterministic solution; associated with this, we observe only a small variation in the functional. Thus, an error caused by lack of information in obtaining the initial condition generated small variations in cost and did not result in drastic changes for the decision maker.

\section{B. Analysis of the interval problem (8)}

For the interval problem (8), the behaviors of the interval state variable $X$ and interval control variable $U$ followed the same trajectory as that of the deterministic solution after the thirteenth day. Thus, for the state variable $X$ (preys) and with the introduction of predators $U$, there was no large variation in comparison with the deterministic solution after the thirteenth day. However, in the initial periods, the introduction of predators $U$ presented a large variation, with direct implications for agricultural practice of pest control.
We emphasize the large variation of the interval state variable $Y$, which represents the variation of the predators (Figure 5). For the third period, we obtained a variation of $5.1270 \times 10^{3}$ up to $1.4565 \times$ $10^{4}$ corresponding to the $Y$ optimal interval state given by the interval $\left[5.1270 \times 10^{3}, 1.4565 \times 10^{4}\right]$. For this variable, we obtained an approximation of the extremes of the interval, which represents the interval solution, to the deterministic solution after the fifteenth day. Furthermore, the problem presents a large variation in the optimal interval cost.

Finally, we can conclude that the facts described above will certainly influence the company's decision making.

\section{Conclusion}

In Section III. we perceive that the optimal interval state $X$ was approximately 2000 in problems (6) and (8). The optimal interval state $Y$ (predators) also approximated the desired value. The optimal interval control tends to the value of 16 wasps per day for the two situations.

These values approximated the results presented in [5]. Botelho and Macedo in [5] show that the application of control in the population of caterpillars in the State of São Paulo - Brazil utilizing the parasitoid Cotesia Flavipes stabilized the number of caterpillars to $x=1900$ per hectare. The number of wasps per hectare stabilized to $y=1423$ with the average rate of introduction of 16.4 wasps per day.

Thus, considering the deterministic or interval problem, the values that represent the solution to the problem are near the desired values and in accordance with the actual situation practiced in the State of São Paulo.

For the implementation of biological control in practice, the simulation results show us that we should introduce a daily number of predators (Cotesia Flavipes) in the tillage, and this number should be contained in the interval solution. We remark that inserting large numbers of predators does not necessarily guarantee a higher cost compared to the costs that are contained in the optimal interval cost and does not necessarily guarantee 
José Renato Campos et al., Biological control of sugarcane caterpillar ...

a control of the infestation in a shorter time, although this is a likely outcome for both interval problems studied. We only know that independent of the number of predators inserted in tillage, and because this number of predators is contained in the interval solution, we can control the infestation with a cost contained in the optimal interval cost state and control contained in the optimal interval state and optimal interval control, respectively. Furthermore, the daily number of predators inserted in tillage corresponds to the difference, in absolute value, between the number of predators inserted the previous day and the number that will be inserted the day after.

\section{ACKNOWLEDGMENT}

The authors wish to express their sincere thanks to the referees for valuable suggestions that improved the manuscript.

The authors also thank the CAPES - Coordination for the Improvement of Higher Education Personnel. The author Edvaldo Assunção was partially supported by the $\mathrm{CNPq}-$ Brazilian National Council for Scientific and Technological Development - under Grant number 300703/20139. The author Geraldo Nunes Silva was partially supported by the São Paulo State Research Foundation (FAPESP - CEPID) under Grant number 2013/07375-0.

\section{REFERENCES}

[1] AGRIANUAL 2012: anuário da agricultura brasileira, São Paulo: FNP, 2012.

[2] R. E. Bellman, Dynamic programming, Princeton: University Press, 1957.

[3] D. P. Bertsekas, Dynamic programming and optimal control, vol. I, Belmont Massachusetts: Athena Scientific, 1995.

[4] D. P. Bertsekas, Dynamic programming and stochastic control, New York: Academic Press, 1976.

[5] P. S. M. Botelho and N. Macedo, Cotesia Flavipes para o controle de Diatraea Sacharalis, In: J. R. P. Parra, P. S. M. Botelho, B. S. C. Ferreira and J. M. S. Bento, Controle biológico no Brasil: parasitóides e predadores, São Paulo: Manole, 409-447, 2002.

[6] S. Boyd, L. E. Ghaoui, E. Feron and V. Balakrishnan, Linear matrix inequalities in system and control theory, Philadelphia: SIAM, 1994.
[7] J. R. Campos, Controle ótimo da lagarta da canade-açúcar utilizando modelos linearizados e funcional quadrático: uma resolução usando programação dinâmica, Universitas, 3 (1): 241-252, 2007.

[8] E. Cerdá, Optimización dinámica, Madri: Pearson Educación, 2001.

[9] Y. Chalco-Cano, W. A. Lodwick and B. Bede, Single level constraint interval arithmetic, Fuzzy Sets and Systems, 2014. http://dx.doi.org/10.1016/j.fss.2014.06.017 http://dx.doi.org/10.1016/j.fss.2014.06.017

[10] M. M. Diniz and R. C. Bassanezi, Problema de controle ótimo com equações de estado p-fuzzy: programação dinâmica, Biomatemática, 23: 33-42, 2013.

[11] C. J. Doyle, A review of the use of models of weed control in integrated crop protection, Agriculture, Ecosystems and Environment, 64: 165-172, 1997. http://dx.doi.org/10.1016/S0167-8809(97)00035-2

[12] D. Filev and A. Plamen, Fuzzy optimal control, Fuzzy Sets and Systems, 47: 151-156, 1992. http://dx.doi.org/10.1016/0165-0114(92)90172-Z

[13] J. C. Geromel, P. L. D. Peres and S. R. Souza, $H_{2}$ guaranteed cost control for uncertain continuous-time linear systems, Systems \& Control Letters, 19: 23-27, 1992. http://dx.doi.org/10.1016/0167-6911(92)90035-Q

[14] J. C. Geromel, P. L. D. Peres and S. R. Souza, $H_{\infty}$ control of discrete-time uncertain systems, IEEE Transactions on Automatic control, 39: 1072-1075, 1994. http://dx.doi.org/10.1109/9.284896

[15] R. Jones and O. J. Cacho, A dynamic optimisation model of weed control, Agricultural and Resource Economics, 1: 1-17, 2000.

[16] J. O. S. Kennedy, Dynamic programming: applications to Agriculture and natural resources, New York: Elsevier, 1986.

[17] K. Kobayashi and K. Hiraishi, Analysis and control of hybrid systems with parameter uncertainty based on interval methods, American Control Conference, 36323637, 2009. http://dx.doi.org/10.1109/ACC.2009.5159932

[18] U. A. S. Leal, Incerteza intervalar em otimização $e$ controle, 2015, 163 f. Tese (Doutorado em Matemática), Universidade Estadual Paulista, São José do Rio Preto, 2015.

[19] U. A. S. Leal, G. N. Silva and D. Karam, Otimização dinâmica multiobjetivo da aplicação de herbicida considerando a resistência de plantas daninhas, Biomatemática, 22: 1-16, 2012.

[20] B. Li, R. Chiong and M. Lin, A two-layer optimization framework for UAV path planning with interval uncertainties, Computational Intelligence in Production and Logistics Systems, 120-127, 2014.

[21] W. A. Lodwick, Constrained interval arithmetic, CCM Report 138, 1999. http://dx.doi.org/10.1109/CIPLS.2014.7007170

[22] W. A. Lodwick, Interval and fuzzy analysis: an unified approach, In: Advances in Imagining and Electronic Physics, Academic Press, 148: 75-192, 2007. 
José Renato Campos et al., Biological control of sugarcane caterpillar ...

[23] W. A. Lodwick and O. A. Jenkins, Constrained interval and interval spaces, Soft Computing, 17: 1393-1402, 2013. http://dx.doi.org/10.1007/s00500-013-1006-x

[24] R. E. Moore, R. B. Kearfott and M. J. Cloud, Introduction to interval analysis, Philadelphia: SIAM - Society for Industrial and Applied Mathematics, 2009.

[25] L. H. Monteiro, Sistemas dinâmicos, São Paulo: Editora Livraria da Física, 2002.

[26] K. Ogata, Engenharia de controle moderno, Rio de Janeiro: LTC, 1998.

[27] J. R. P. Parra, P. S. M. Botelho, B. S. C. Ferreira and J. M. S. Bento, Controle biológico no Brasil: parasitóides e predadores, So Paulo: Manole, 2002.

[28] C. M. Pereira, M. S. Cecconello and R. C. Bassanezi, Controle ótimo em sistemas baseados em regras fuzzy, Biomatemática, 23: 147-167, 2013.

[29] L. S. Pontryagin, Optimal regulation processes, In: Proceedings of International Congresses of Mathematicians, 1958.

[30] L. S. Pontryagin, V. G. Boltyansky, P. V. Gamkrelidze and E. F. Mischenko, The mathematical theory of optimal processes, New York: Interscience Publishers, 1962.

[31] M. Rafikov and P. A. P. Borges Alguns aspectos do controle em um modelo dinâmico de objetos de produção de interações conforme uma funcional quadrática, Análise Econômica, 19: 103-121, 1993.

[32] A. Rauh, J. Minisini and E. P. Hofer, Interval techniques for design of optimal and robust control strategies,
Proceedings of the Twelfth GAMM-IMACS International Symposium on Scientific Computing, Computer Arithmetic and Validated Numerics, 1-9, 2006. http://dx.doi.org/10.1109/SCAN.2006.27

[33] S. V. Segato, A. S. Pinto, E. Jendiroba and J. C. M. Nóbrega Atualização em produção de cana-de-açúcar, Piracicaba: Livroceres, 2006.

[34] Y. P. Shih and C. J. Chen On the weighting factores of the quadratic criterion in optimal control, International Journal of Control, 19: 947-955, 1974. http://dx.doi.org/10.1080/00207177408932688

[35] F. C. Silva and A. F. Bergamasco, Levantamento de modelos matemáticos descritos para a cultura da canade-açúcar, Embrapa Informática Agropecuária, 2001.

[36] H. A. Taha, Pesquisa operacional, São Paulo: Pearson Prentice Hall, 2008.

[37] A. M. Tusset and M. Rafikov, Controle ótimo da lagarta da cana-de-açúcar, utilizando modelos linearizados e funcional quadrático, In: Anais do $3^{\circ}$ Congresso Temático de Dinâmica e Controle da SBMAC, Ilha Solteira, 1572-1577, 2004.

[38] UNICA: União da Indústria de Cana-de-açúcar. Available at: http://unica.com.br .

[39] L. Yu, J. M. Xu and Q. L. Han, Optimal guaranteed cost control of linear uncertain systems with input constraints, Proceedings of the Fifth World Congress on Intelligent Control and Automation, 553-557, 2004.

http://dx.doi.org/10.1109/WCICA.2004.1340636 